\title{
Anatomy and Physiology for the Abdominal Aortic Aneurysm Repair
}

\author{
Takuya Matsumoto, MD, PhD, FACS
}

In 2006, commercially produced endovascular aneurysm repair (EVAR) devices were approved by the Japanese Ministry of Health, Labour and Welfare, and their cost began to be covered by Japanese medical insurance. Meanwhile, the number of juxtarenal abdominal aortic aneurysms (AAA) to need the suprarenal clamp are increasing and the number of infra-renal AAAs are decreasing for open repair. In this era when EVAR has been growing rapidly for 11 years, it is a good opportunity to learn the surgical repair of AAA. I review the basic and advanced anatomy and physiology concepts which are needed for abdominal aortic repair, which are the proximal site (exposure of the proximal site, variation of renal arteries, variation of inferior vena cava and left renal vein, arcade of visceral branches of abdominal aorta, and coeliac plexus) and distal site (iliac artery, superior hypogastric plexus, ureter, inferior mesenteric artery, and lumbar arteries) separately. (This is a translation of Jpn J Vasc Surg 2019; 28: 173-177.)

Keywords: abdominal aortic aneurysm, anatomy and physiology, thoracoabdominal approach

\section{Introduction}

The recent introduction of stent grafting has diminished opportunities for surgeons-in-training to perform resection and reconstruction using a vascular graft for abdomi-

Department of Vascular Surgery, Graduate School of Medical Sciences, International University of Health and Welfare, Narita, Chiba, Japan

Received: June 13, 2019; Accepted: June 14, 2019

Corresponding author: Takuya Matsumoto, MD, PhD. Department of Vascular Surgery, Graduate School of Medical Sciences, International University of Health and Welfare, 4-3 Kouzunomori, Narita, Chiba 286-8686, Japan

Tel: +81-476-20-7701, Fax: +81-476-20-7702

E-mail: tak@iuhw.ac.jp

This is a translation of Jpn J Vasc Surg 2019; 28: 173-177.

(cc) BY-NC-SA (C2019 The Editorial Committee of Annals of Vascular Diseases. This article is distributed under the terms of the Creative Commons Attribution License, which permits use, distribution, and reproduction in any medium, provided the credit of the original work, a link to the license, and indication of any change are properly given, and the original work is not used for commercial purposes. Remixed or transformed contributions must be distributed under the same license as the original. nal aortic aneurysm (AAA). On the contrary, for patients in whom performing stent grafting is arduous, such as pararenal, juxtarenal, and suprarenal AAA with a short landing zone, open surgery is often performed, which requires reexamination of the anatomy and physiology required for surgery. Needless to say, it is important to expose the proximal neck, and in the event of a rupture, the proximal neck is sought along the aneurysmal wall to avoid secondary damage to surrounding organs. However, the difficulty in manipulation for dissection is enhanced for large aneurysms and short infrarenal necks. This instructional report outlines the anatomical and physiological background required for AAA repair.

\section{The Abdominal Aorta and Its Branches}

The abdominal aorta commences at the level of the Th12 vertebra via the aortic hiatus at the diaphragm. At the Th12 and L1 levels, the celiac artery branches anteriorly from the aorta, and immediately below which the largest visceral artery, that is, the superior mesenteric artery, branches laterally. At the L1-L2 level, approximately $1-2 \mathrm{~cm}$ further below, the left and right renal arteries branch off. At the L3 level, the inferior mesenteric artery branches off ventrally or in an inferior left direction. It branches into the left and right common iliac arteries at the L4 level (Fig. 1).

\section{The Proximal Anatomy and Physiology}

\section{Proximal exposure}

To expose the neck of infrarenal AAAs, the left renal vein is defined as a landmark and the retroperitoneum is sectioned between the duodenum and the inferior mesenteric vein. Generally, the renal artery can be seen dorsal to the superior border of the left renal vein. If the neck is short, the exposure of the left and right renal arteries, and the aorta below the renal arteries, is facilitated by sufficiently mobilizing the left renal vein (Fig. 2a) or sectioning it (Fig. $2 b$ ). To displace the left renal vein, the adrenal vein flowing into the left renal vein and the left testicular/ovarian vein are ligated and sectioned. When sectioning the left renal vein, the adrenal vein and left testicular/ovarian vein 

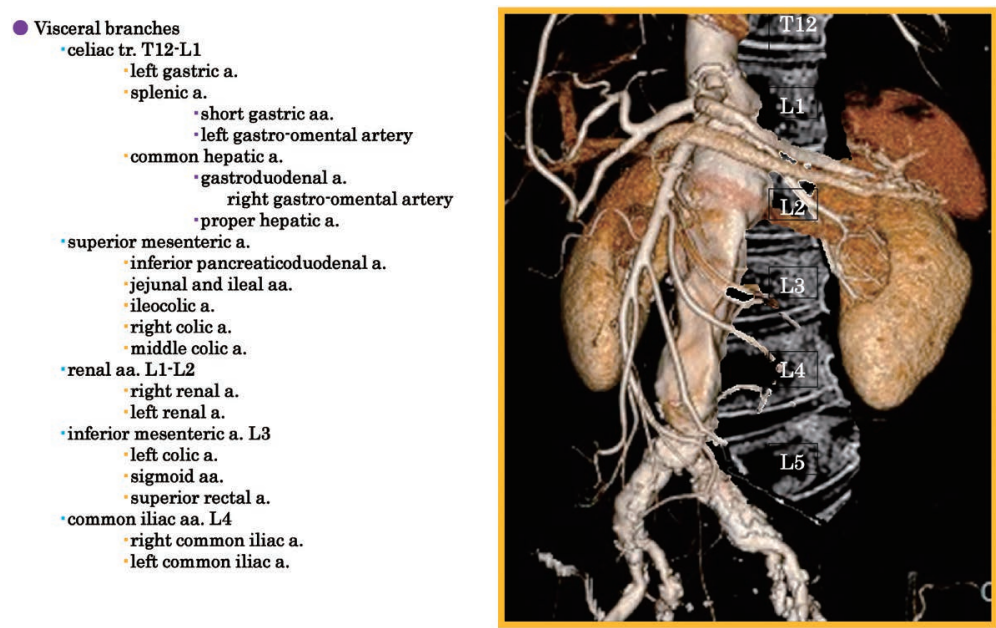

Fig. 1 Visceral branches of abdominal aorta.

3DCT shows visceral branches of the juxtarenal abdominal aortic aneurysm.
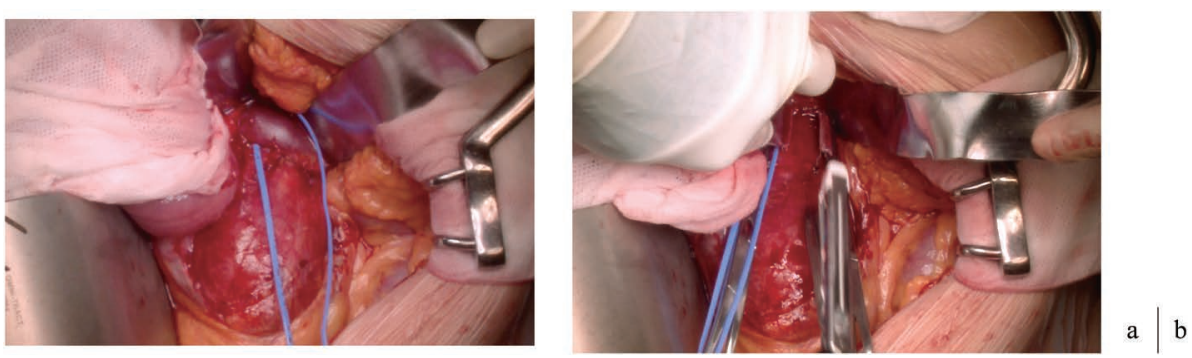

Fig. 2 (a) Exposure of left renal vein. (b) Resection of left renal vein.

This picture shows the resection of left renal vein which is cut as close to the vena cava as possible for the juxtarenal AAAs using the Endo GIA.

turn into important collateral circulation, and, therefore, to preserve these veins, they should be dissected as close as possible to the inferior vena cava (Fig. 2b). For AAA repair, the dissection of the renal vein can reduce postoperative renal function; however, this improves in 2-6 weeks ${ }^{1)}$ and has no long-term impact on renal functioning. ${ }^{2}$

Due to the difficulty in approaching the aorta from above the superior mesenteric and celiac arteries, sectioning the lesser omentum between the stomach and liver allows the exposure of the aorta above the celiac artery. Thereafter, an incision is made to the retroperitoneum at the anterior surface of the crura of the diaphragm. Upon exposing the crura of the diaphragm along the muscle fibers, the abdominal aorta is bluntly dissected just beneath the diaphragm, pinched between the fingers, and subsequently clamped.

Severe atheromatous changes in the abdominal aorta above the renal artery and severe adhesions caused by an infected aneurysm can make clamping just above the renal artery difficult, posing a challenge for dissection. If the need arises, clamping of the aorta between the superior mesenteric and celiac arteries, or clamping of the latter two arteries can be achieved using the left medial visceral

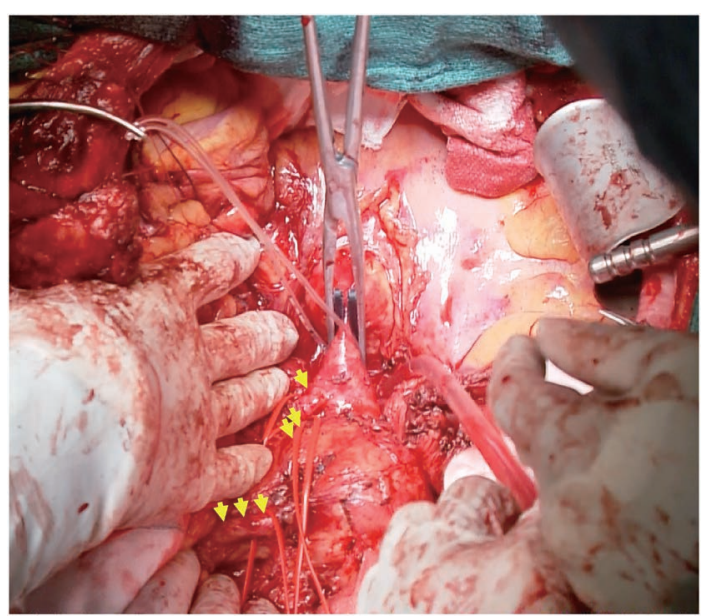

Fig. 3 Thoracoabdominal approach.

A single arrow identifies the celiac artery, two arrows identifies the superior mesenteric artery, three arrows identifies the left renal artery. Celiac artery $(\downarrow)$, superior mesenteric artery ( $\downarrow \downarrow)$, left renal artery ( $\downarrow \downarrow)$.

rotation method through an abdominal midline incision to expose the entire abdominal aorta by dislocating the left colon and spleen, or using the thoracoabdominal ap- 
proach by thoracolaparotomy (Fig. 3). While this can facilitate the exposure of the superior mesenteric and celiac arteries, this procedure is more invasive.

\section{Renal artery variations}

According to Ozkan et al., ${ }^{3)}$ one right renal artery was observed in $83 \%$ of the patients; however, no right renal arteries were observed in $0.8 \%, 2$ were observed in $15 \%$, 3 in $1 \%$, and 4 in $0 \%$. Along the same lines, 1 left renal artery was observed in $86 \%$ of the patients, with no left renal artery observed in $0.7 \%, 2$ in $12 \%, 3$ in $0.7 \%$, and 4 in $0.2 \%$. Furthermore, in $98 \%$ of the patients, the right main renal artery originated between the upper margin of the $\mathrm{L} 1$ and the lower margin of the $\mathrm{L} 2$ vertebrae, originating from the $\mathrm{L} 1$ in $43 \%, \mathrm{~L} 1-\mathrm{L} 2$ in $23 \%$, and $\mathrm{L} 2$ in $32 \%$; whereas on the left side, in $97 \%$ of the patients the main renal artery originated between the upper margin of the $\mathrm{L} 1$ and lower margin of the L2 vertebrae, originating from the $\mathrm{L} 1$ in $37 \%, \mathrm{~L} 1-\mathrm{L} 2$ in $22 \%$, and $\mathrm{L} 2$ in $38 \%$, indicating that in most patients the left and right main renal arteries originated from approximately the same level. ${ }^{4}$ The right renal artery often originated from a higher aortic level than the left side, in cases where the origin location differed. No major clinical problems are expected in the event that the accessory renal arteries are obstructed during stent grafting and in patients with mild renal insufficiency ${ }^{5,6}$; however, it has been reported that renal function is reduced even in patients with normal preoperative renal function. ${ }^{7)}$ Furthermore, in the event of a horseshoe kidney, $60 \%$ of patients exhibit abnormal branching of the renal arteries, ${ }^{8}$ and the accessory renal arteries often originate from aneurysms. If such aneurysms are $2 \mathrm{~mm}$ or greater in size, the arteries should be reconstructed as a general rule..$^{9,10)}$

\section{Inferior vena cava variations}

The inferior vena cava is normally located on the right side of the abdominal aorta and does not require dissection. However, a damaged vein can cause massive bleeding, and, therefore, in the event of proximal neck dissection, this anomaly demands extreme caution. Variations of the inferior vena cava arising during its embryologic development include left inferior vena cava in $0.2 \%-0.5 \%$, and the duplication of the inferior vena cava in $0.2 \%-0.3 \%$ of the general population. Furthermore, the incidence of retroaortic left renal vein is approximately $1.7 \%-8.7 \%$, and in some cases, a circumaortic renal collar is observed. ${ }^{11)}$

\section{Arcade of each branch}

The clamping site for AAA requiring suprarenal artery clamping is above the renal artery in $54 \%$ of the patients, above the superior mesenteric artery in $30 \%$, and above the celiac artery in $16 \%{ }^{12)}$ In simple clamping, $2.9 \%$ of patients required intestinal resection, ${ }^{12)}$ and I believe that the confirmation of each branch arcade using the balloon occlusion test on angiography is important in the event of superior mesenteric artery-aorta clamping. The permissible ischemia time is $10-20 \mathrm{~min}$ for celiac artery clamping, and if the visceral ischemia time exceeds $32 \mathrm{~min}$, it is considered as a prognostic factor of early death. ${ }^{13)}$ The anticipation of prolonged clamping necessitates superior mesenteric artery blood transmission. The celiac artery has three branches including the left gastric artery, common hepatic artery, and splenic artery; the junction of the celiac artery and superior mesenteric artery systems is formed via the dorsal pancreatic artery along with the superior and inferior pancreaticoduodenal arteries (pancreatic head arcade). ${ }^{14)}$ At the junction of the celiac and inferior mesenteric arteries, the dorsal pancreatic artery originating from the celiac artery, along with the inferior mesenteric artery, connects to the trunk of the left colic artery branching from the inferior mesenteric artery.

\section{Superior mesenteric plexus}

To expose the celiac artery root by transperitoneal laparotomy, the celiac ganglia need to be sectioned. The removal of this celiac plexus can cause severe diarrhea, making postoperative management difficult and requiring strict management using various anti-diarrhea agents. Diarrhea is due to digestion absorption disorders accompanied by lymph duct congestion ${ }^{15)}$ and increased small intestine motility caused by autonomic nerve blockage caused during outpatient care. ${ }^{16)}$

\section{Distal Anatomy and Physiology}

\section{Iliac artery}

The common iliac artery runs for $3-4 \mathrm{~cm}$, after which it branches into internal and external iliac arteries. Furthermore, the iliac veins are located medial and posterior to the iliac artery, and the left common iliac vein crosses dorsal to the right common iliac artery and flows into the right common iliac vein at the L5 level, which flows into the inferior vena cava. Caution should be exercised when dissecting the whole circumference of the right common iliac artery at its root. The internal iliac artery divides into the anterior and posterior divisions, with the former possessing several arterial branches including the inferior gluteal and internal pudendal arteries, while the posterior division branches off into the superior gluteal and iliolumbar arteries. The internal iliac artery is exposed for a length of $1-6 \mathrm{~cm}$ up to its bifurcation; in many instances, if the internal iliac artery is long, the common iliac artery is short, whereas the opposite is also frequently observed. The internal iliac artery is involved in various types of physiological functions, including sexual dysfunction, but- 
tock claudication, intestinal ischemia, and paraplegia. In sexual dysfunction, lack of sufficient blood flow in a short period to the deep artery of the penis can cause erectile dysfunction. Therefore, blood flow should be preserved from at least one side of the internal iliac arteries.

It has been suggested that in gluteal claudication expressed according to the systemic pressure ratio following Y-grafting, on measuring the internal iliac artery stump pressure while the inferior mesenteric artery and bilateral internal iliac arteries are clamped, the internal iliac artery pressure/systemic pressure ratio can be less than 0.5 ; it has also been suggested that ischemic enteritis can occur when the internal iliac artery pressure/systemic pressure ratio is approximately $0.3 .{ }^{17)}$ Therefore, Sai et al. recommended the requirement of internal iliac artery reconstruction because internal iliac artery pressure/systemic pressure ratio of $<0.5$ can lead to reduced quality of life owing to ischemic colitis, gluteal claudication, and sexual dysfunction. ${ }^{17)}$ Paraplegia following AAA repair is extremely rare. If it does occur, the onset is either immediately after surgery or may be delayed, the mechanisms of which are said to differ. Onset immediately after surgery is postulated to be caused by low oxygen injury caused by reduced blood flow to the spinal cord, whereas delayed onset results from localized reduced blood flow caused by spinal cord edema. ${ }^{18)}$ Reduced blood flow from collateral circulation to the spinal artery may be caused by vascular insufficiency, which is attributed to the absence of revascularization of the inferior mesenteric and internal iliac arteries. According to the collateral network concept proposed by Griepp RB and Griepp EB, the spinal cord is supplied with blood from many collateral pathways from the subclavian to the internal iliac arteries; therefore, the preservation of these arteries and the maintenance of perioperative blood pressure are important for the prevention of paraplegia. ${ }^{19,20)}$

\section{The superior hypogastric plexus}

The superior hypogastric plexus is located in the space extending from the lower margin of the inferior mesenteric artery root to the bifurcation of the aorta. The second to the fourth lumbar splanchnic nerves join the aortic plexus, located anterior to the fifth lumbar vertebral body to the first sacral vertebral body, and run in the middle of the left common iliac artery. Because they contribute to ejaculation, their preservation is recommended. Sexual dysfunction following AAA repair is broadly divided into retrograde ejaculation caused by injury of these nerves and blood flow-related erectile dysfunction caused by the interruption of blood flow from the bilateral internal iliac arteries.

\section{Ureter}

The ureter runs above the bifurcation of the internal and external iliac arteries; however, when exposing the iliac arteries, adhesions can render identification difficult. This can be overcome by placing a ureteral stent preoperatively as a landmark. Previously, hydronephrosis was attributed to the ureter being positioned dorsally to the graft. However, cases of hydronephrosis onset despite the graft being placed dorsal to the ureter have been reported. Causes other than the positional relationship of the graft and the ureter include retroperitoneal inflammation, edema, retroperitoneal hematoma, retroperitoneal fibrosis, tissue damage, graft infection, and pseudoaneurysm. ${ }^{21)}$ During surgery, it is vital that the ureter is not dissected and that the graft and ureter do not come into direct contact.

\section{The inferior mesenteric artery}

The marginal artery of Drummond (Drummond's marginal artery) at the splenic flexure lies at the junction of the superior mesenteric and inferior mesenteric arteries. However, medial to the marginal artery, the arch of Riolan can be seen connecting the two arteries. This arterial arch branches directly from the superior mesenteric artery, as well as from the middle colic artery, and inferior pancreaticoduodenal artery, and runs along the margin of the intestinal tract from the superior margin of the duodenojejunal flexure, and along with the inferior mesenteric artery, connects to the main trunk of the left colic artery originating from the inferior mesenteric artery. ${ }^{14)}$ Normally, the ligation of the inferior mesenteric artery does not contribute to intestinal necrosis ${ }^{22}$; , however, following colectomy, and in the event of obstruction or stenosis of the celiac and root of the superior mesenteric artery, the collateral circulation should be verified as unnecessary ligation of the inferior mesenteric artery may cause intestinal necrosis.

\section{The lumbar arteries}

The lumbar arteries are paired lateral branches originating from the abdominal aorta toward the lumbar spine. Normally, the lumbar arteries are present as four pairs of posterolateral branches of the abdominal aorta at the L1-L4 level. They run anterior to the lumbar vertebrae and disappear deep into the greater psoas muscle. All lumbar arteries on the right side run along the posterior wall of the inferior vena cava. The first and second lumbar arteries are covered by the crura of the lumbar part of the diaphragm. Furthermore, the right-sided lumbar arteries are slightly longer than the left-sided counterparts. The lumbar quadrate muscle is cut transversally to expose the area between the transverse abdominal muscle and internal oblique muscle of abdomen to enable reaching the inferior artery anteriorly, the posterior intercostal artery 
superiorly, with the iliolumbar artery and circumflex iliac artery inferiorly. In some instances, there is a small fifth pair of lumbar arteries originating from the middle sacral artery. When performing suture ligation from within the aneurysm for the intercostal arteries, caution should be exercised in the same way as when suturing the esophagus. For the lumbar arteries, due care is warranted to avoid erroneous placement of sutures in the small intestine. In the event of extra-aneurysmal ligation, due care should be taken to prevent damage to the lumbar arteries that run parallel. On occasion, during dissection, the root may be removed from the inferior vena cava, making it necessary to repair the inferior vena cava by suturing.

\section{Conclusion}

The intent of this instructional review was to provide details of the anatomy and physiology required for AAA repair in a thorough fashion to enable surgical treatment for AAA with few complications.

\section{Disclosure Statement}

There are no conflicts of interest to declare.

\section{Additional Note}

The salient features of this report were presented at the 28th Educational Seminar of the Japanese Society for Vascular Surgery (October 27, 2018, Hiroshima, Japan).

\section{References}

1) Mehta T, Wade RG, Clarke JM. Is it safe to ligate the left renal vein during open abdominal aortic aneurysm repair? Ann Vasc Surg 2010; 24: 758-61.

2) Samson RH, Lepore MR Jr, Showalter DP, et al. Long-term safety of left renal vein division and ligation to expedite complex abdominal aortic surgery. J Vasc Surg 2009; 50: 500-4; discussion, 504.

3) Ozkan U, Oğuzkurt L, Tercan F, et al. Renal artery origins and variations: angiographic evaluation of 855 consecutive patients. Diagn Interv Radiol 2006; 12: 183-6.

4) Aubert J, Koumare K. Variations of origin of the renal artery: a review covering 403 aortographies. Eur Urol 1975; 1: 1828.

5) Abu Bakr N, Torsello G, Pitoulias GA, et al. Preservation of clinically relevant accessory renal arteries in infrarenal AAA patients with adequate proximal landing zones undergoing EVAR. J Endovasc Ther 2016; 23: 314-20.

6) Karmacharya J, Parmer SS, Antezana JN, et al. Outcomes of accessory renal artery occlusion during endovascular aneurysm repair. J Vasc Surg 2006; 43: 8-13.

7) Sadeghi-Azandaryani M, Zimmermann H, Korten I, et al.
Altered renal functions in patients with occlusion of an accessory renal artery after endovascular stenting of an infrarenal aneurysm. J Vasc Surg 2017; 65: 635-42.

8) Connelly TL, McKinnon W, Smith RB III, et al. Abdominal aortic surgery and horseshoe kidney. Arch Surg 1980; 115: 1459-63.

9) O'Hara PJ, Hakaim AG, Hertzer NR, et al. Surgical management of aortic aneurysm and coexistent horseshoe kidney: review of a 31-year experience. J Vasc Surg 1993; 17: 940-7.

10) Cohn LH, Stoney RJ, Wylie EJ. Abdominal aortic aneurysm and horseshoe kidney. Ann Surg 1969; 170: 870-4.

11) Bass JE, Redwine MD, Kramer LA, et al. Spectrum of congenital anomalies of the inferior vena cava: cross-sectional imaging findings. Radiographics 2000; 20: 639-52.

12) Wartman SM, Woo K, Yaeger A, et al. Outcomes after abdominal aortic aneurysm repair requiring a suprarenal crossclamp. J Vasc Surg 2014; 60: 893-9.

13) Furuya T. Open repair for pararenal abdominal aortic aneurysm: the strategy and pitfalls for safe surgery. Nihon Geka Gakkai Zasshi 2011; 112: 17-21. (in Japanese)

14) Matsui O. Vascular anatomy of the celiac artery, superior mesenteric artery (SMA), and inferior mesenteric artery (IMA) for diagnostic imaging and Interventional radiology. Japan-Germany medical reports 2006; 51: 54-77. (in Japanese)

15) Isobe T. Experimental study of absorptional function after lymphatic dissection around the root of superior mesenteric artery. Japanese Journal of Gastroenterological Surgery 1989; 22: 806-13. (in Japanese)

16) Takano N. An experimental study of intestinal absorptive function after resection of celiac ganglion. Japanese Journal of Gastroenterological Surgery 1988; 21: 2271-7. (in Japanese)

17) Sai K, Ichik M, Sugahara $H$, et al. Indication of internal iliac artery reconstruction with QOL in focus-Pelvic blood flow evaluation by measuring internal iliac artery stump pressure. The Journal of Japanese College of Angiology 2005; 45: 347-51. (in Japanese)

18) Crawford ES, Mizrahi EM, Hess KR, et al. The impact of distal aortic perfusion and somatosensory evoked potential monitoring on prevention of paraplegia after aortic aneurysm operation. J Thorac Cardiovasc Surg 1988; 95: 357-67.

19) Griepp RB, Griepp EB. Spinal cord perfusion and protection during descending thoracic and thoracoabdominal aortic surgery: the collateral network concept. Ann Thorac Surg 2007; 83: S865-9; discussion, S890-2.

20) Asano T. Basics of spinal vascular anatomy and spinal angiography. Available from: http://nnac.umin.jp/nnac/di2huipu roguramu_files/Asano.pdf (Accessed: July 17, 2017) (in Japanese)

21) Sant GR, Heaney JA, Parkhurst EC, et al. Obstructive uropathy-a potentially serious complication of reconstructive vascular surgery. J Urol 1983; 129: 16-22.

22) Furuya T, Tanaka N, Nobori N, et al. Is inferior mesenteric artery reconstruction necessary in abdominal aortic aneurysmectomy? A study of ruptured and non-ruptured abdominal aortic aneurysm with regard to pelvic circulation. Japanese Journal of Vascular Surgery 2001; 10: 1-7. (in Japanese) 